\title{
The INFLUENCE OF CUTTING EdGE RADIUS ON FORCE LOAD WHEN MILLING INCONEL 718
}

\author{
Hronek Ondřej, Zetek Miroslav \& Bakša Tomáš
}
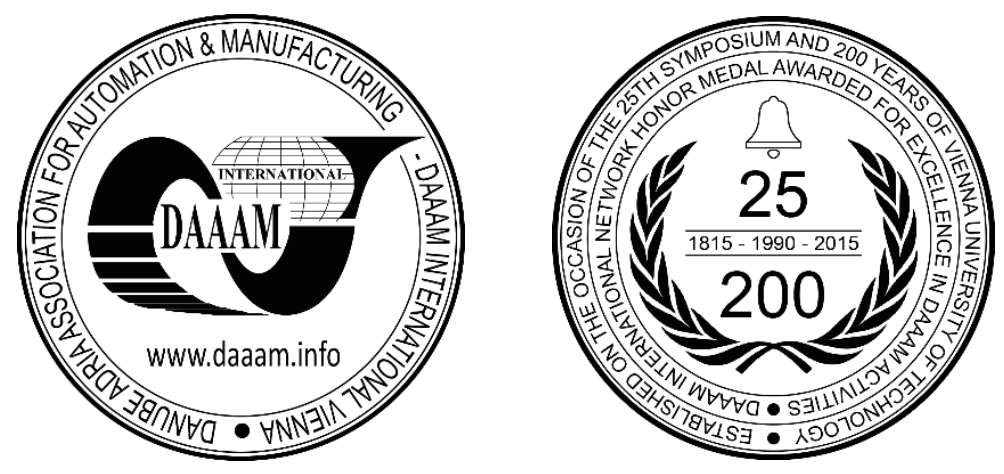

This Publication has to be referred as: Hronek, O[ndrej]; Zetek, M[iroslav] \& Baksa, T[omas] (2018). The Influence of Cutting Edge Radius on Force Load when Milling Inconel 718, Proceedings of the 29th DAAAM International Symposium, pp.0822-0828, B. Katalinic (Ed.), Published by DAAAM International, ISBN 978-3-902734-20-4, ISSN 1726-9679, Vienna, Austria

DOI: $10.2507 / 29$ th.daaam.proceedings.119

\begin{abstract}
The cutting edge radius of a cutting tool plays a significant role in its durability. Durability is even more important when heavy duty materials are machined, including nickel alloy Inconel 718. The tool's durability influences the overall efficiency of the cutting process, both technically and economically. This paper investigates the effects of the cutting edge radius on the final cutting forces generated when milling Inconel 718 . The cutting edge radiuses are $15 \mu \mathrm{m} ; 20 \mu \mathrm{m}$ and $25 \mu \mathrm{m}$. A three-component dynamometer was used for measuring the cutting forces, which were measured in the $\mathrm{X}$; $\mathrm{Y}$ and $\mathrm{Z}$ axes. The force measurement was related to the cutting edge wear $\left(\mathrm{VB}_{\mathrm{B}}\right)$ of the clearance surface.
\end{abstract}

Keywords: drag finishing; process conditions; immersion depth; cutting edge modification

\section{Introduction}

A state of tension in the machined layer is created when a cutting tool enters the workpiece material. This state causes chip formation after reaching a certain amount of mechanical and thermal energy. The process of chip formation is accompanied by plastic and elastic deformations. Force loads occur during the machining process.Machining force is an important quantity. This force is not constant, it depends on cutting tool wear, machining time and tool geometry. [1] The physical law of action and reaction applies during machining, so the state of stress in the machined material is also caused by cutting resistance $\mathrm{R}$. The cutting force is equal to the cutting resistance. For the equilibrium condition, the following applies [2]:

$$
F=-R
$$

F- cutting force [N],

$\mathrm{R}$ - cutting resistance $[\mathrm{N}]$.

The cutting force generated during machining is a dynamic phenomenon. 
The magnitude of the force varies up to $\pm 20 \%$ during cutting under constant process parameters. [2] The reasons for this fluctuation are inhomogeneous material properties, different energy demands of the chip formation mechanism, wear and micro tearing of the cutting edge. [3] The experiment needs to be repeated to increase the credibility of the measured data.

The measured data is used to analyse the cutting process. These analyses are used to optimize the cutting process. These date make it possible to compare and optimize machining strategies. The measured data serves to design and optimize the cutting angles and the stiffness of the cutting tool. [4] [5] With the cutting forces it is possible to determine the relationship between these forces, cutting edge wear and cutting tool microgeometry, which is the main content of this article.

The force load size is affected by a number of parameters. The basic features include the cutting edge and cutting conditions. These two parameters have a major effect on machining efficiency and chip formation. Also, the cutting edge radius influences the size and direction of the force load. The microgeometry of the cutting edge affects the force load in the direction of the tool feed, and the axial and radial depth of cut. [6] [7] The cutting edge radius can significantly change the force load and final quality of the machined surface.

\subsection{Problem statement}

The cutting process is very demanding despite considerable research and development into new cutting materials, cutting edge geometry, machining strategies and materials which are still being machined in the industry. These hard to machine alloys are especially used in the energetic and aerospace industries, so it is necessary to machine them. The high surface quality and shape accuracy of machined samples plays a very important role in terms of safety. Therefore, the cutting process of these alloys is investigated in order to improve them. [8] The aim of this research is increasing the durability and stability of cutting tools.

\section{The apparatus used in the experiment}

\subsection{Machined material and cutting conditions}

Inconel 718 is the machined material. This nickel alloy was chosen for the reasons outlined in section 1.1 Problem statement. The material specifications are listed in the following table. Before the experiment, the size of the machined workpiece was $290 \times 100 \times 100 \mathrm{~mm}$, but the milled length was just $138 \mathrm{~mm}$.

\begin{tabular}{|c|c|c|c|c|}
\hline Density $\boldsymbol{\rho}$ & $\begin{array}{c}\text { Tensile Strength, } \\
\text { Ultimate }\end{array}$ & $\begin{array}{c}\text { Tensile Strength, } \\
\text { Yield }\end{array}$ & $\begin{array}{c}\text { Elongation at } \\
\text { Break }\end{array}$ & Melting Point \\
\hline$[\mathrm{g} / \mathrm{cc}]$ & {$[\mathrm{MPa}]$} & {$[\mathrm{MPa}]$} & {$[\%]$} & {$\left[{ }^{\circ} \mathrm{C}\right]$} \\
\hline 8.19 & 1375 & 1100 & 25 & $1260-1336$ \\
\hline
\end{tabular}

Table 1. Properties of Inconel 718

Cutting conditions are listed in Table 2. These conditions are based on previous tests. These conditions were constant for all tested cutting tools.

\begin{tabular}{|c|c|c|c|c|}
\hline Cutting Speed $\mathbf{v}_{\mathbf{c}}$ & Feed per Tooth $\mathbf{f}_{\mathbf{z}}$ & Feed Rate vf & $\begin{array}{c}\text { Axial Depth of } \\
\text { Cut } \mathbf{a}_{\mathbf{p}}\end{array}$ & $\begin{array}{c}\text { Radial Depth of } \\
\text { Cut } \mathbf{a}_{\mathbf{e}}\end{array}$ \\
\hline$[\mathrm{m} / \mathrm{min}]$ & {$[-]$} & {$[\mathrm{mm} / \mathrm{min}]$} & {$[\mathrm{mm}]$} & {$[\mathrm{mm}]$} \\
\hline 35 & 0.08 & 222.7 & 3 & 0.5 \\
\hline
\end{tabular}

Table 2. Cutting conditions

\subsection{Cutting tool}

Six cutting tools were ground for this experiment. They were classic end mill tools with two edges. The cutting tool diameter was $8 \mathrm{~mm}$. The cutting tool had a length of $59 \mathrm{~mm}$. The cutting part of the tools had a length of $21 \mathrm{~mm}$. The cutting geometry was the same for all tools: $\gamma_{0}=8^{\circ} ; \alpha_{0}=10^{\circ}$. The helix of the cutting edges $\omega$ had a value of $30^{\circ}$. The cutting tools were clamped $30 \mathrm{~mm}$ into a Tribos polygonal holder during the experimental milling.

The only variable parameter during the experiment was the cutting edge radius. After grinding, the cutting edge radius was $3 \pm 1 \mu \mathrm{m}$. The cutting edge modification followed after cutting edge measurement. Drag finishing technology was selected for the cutting edge modification. Cutting edges of $15 \mu \mathrm{m} ; 20 \mu \mathrm{m}$ and $25 \mu \mathrm{m}$ were the final target of this modification. A two-component process medium of walnut shells and silicon carbide was selected as the finishing medium. After cutting tool modification it was necessary to measure the cutting edges. 
The following figures show a comparison of the ground and modified cutting edge. From the colour spectrum, it is possible to see the increasing cutting edge radius. It is also possible to notice the changes on the chipping and clearance surface quality. Therefore it is necessary to note that the cutting edge modification not only changes the cutting edge radius, but also change the quality of these surfaces.
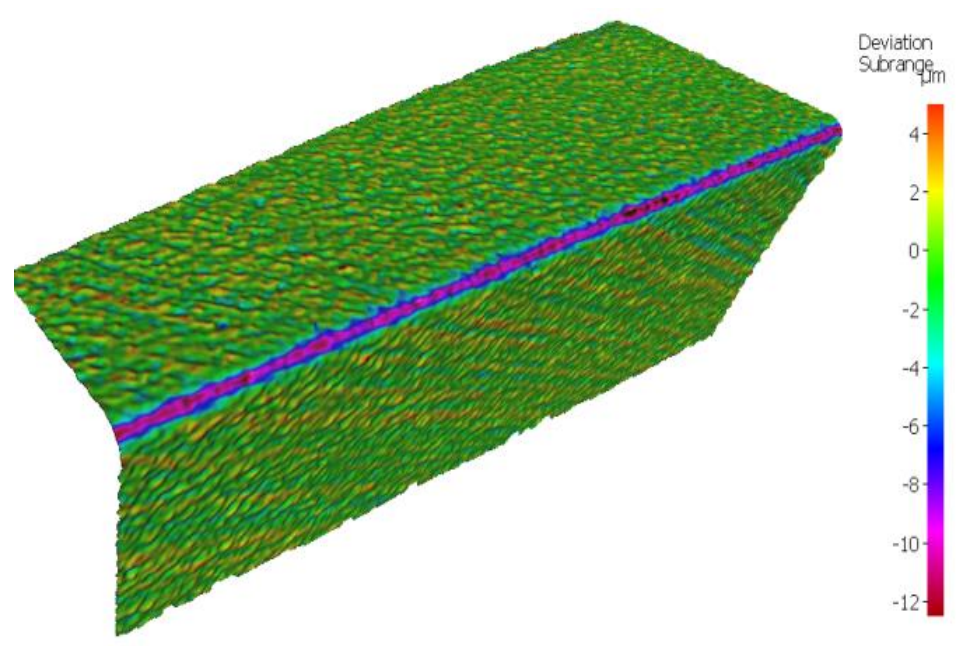

Fig. 1. Cutting edge radius $15 \mu \mathrm{m}$
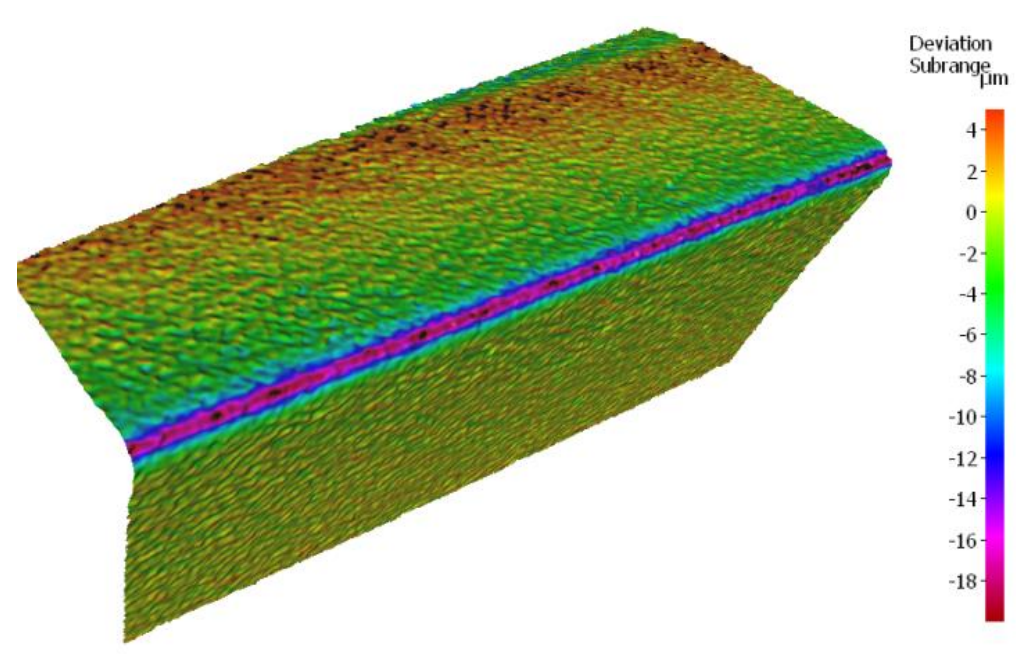

Fig. 2. Cutting edge radius $20 \mu \mathrm{m}$

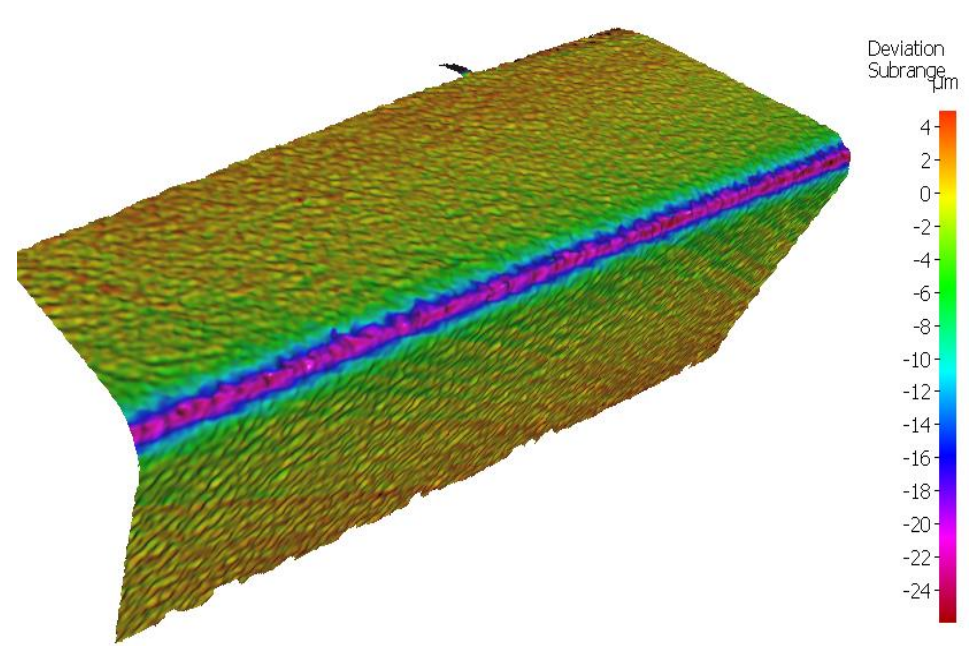

Fig. 3. Cutting edge radius $25 \mu \mathrm{m}$ 


\subsection{Equipment for measuring cutting force}

The force load was the main parameter measured during this experiment. There are a number of methods, both direct and indirect, for measuring force load. In this experiment, a direct force measurement using a dynamometer was chosen. A dynamometer operates on the piezoelectric effect. A static three-component dynamometer Kistler 9255 A was used. This type of dynamometer measures three forces in orthogonal directions. Force Fx acts in the direction of the feed, Fy in the radial depth of cut, and Fz in the cutting tool axis direction. For completeness, the coordinate system of these forces is shown in the following figure. The sampling frequency was set to $1500 \mathrm{~Hz}$. The influences of the cutting edge radius on the cutting forces was measured.

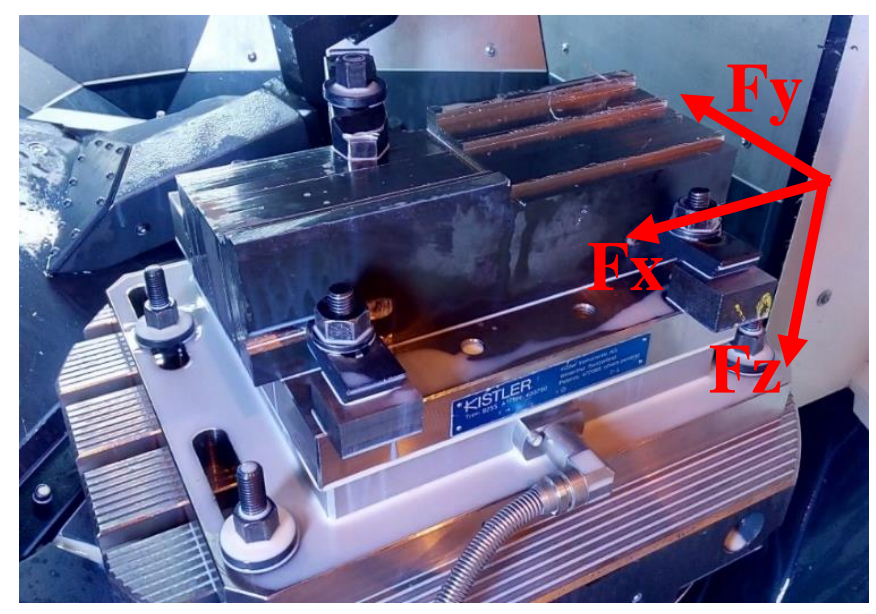

Fig. 4. Cutting force direction

\section{Results and Discussion}

As mentioned earlier, high surface quality of machined parts made from nickel alloys is necessary in the energetic and aerospace industries. The dependence between surface quality, machining forces and cutting edge geometry has already been demonstrated in many research works, therefore this article is focused on the force load in three orthogonal axes.

Forces were measured at three point during the experiment. This measurement depended on the actual wear of the clearance cutting tool surface. Cutting tool wear was measured using a Blickle optical microscope. Cutting edge wear $\mathrm{VB}_{\mathrm{B}}$ was measured. The force load was measured at $\mathrm{VB}_{\mathrm{B}}=0 \mu \mathrm{m} ; \mathrm{VB}_{\mathrm{B}}=100 \mu \mathrm{m}$ and $\mathrm{VB}_{\mathrm{B}}=150 \mu \mathrm{m}$. Limit wear was $\mathrm{VB}_{\mathrm{B}}=150 \mu \mathrm{m}$. The linear type of cutting edge wear was measured for most of the time. Only in a few cases, the wear was not linear, but on the cutting edge there was a notch wear. Already in the first test, tool 1 reached the limit wear on the 9th milling pass. The reason for such a small number of passes was local edge wear, which was higher than the linear wear. The following figure shows the influences of cutting tool microgeometry on the force measurement. The maximum force value was evaluated. The vertical axis gives the force load in Newton's and the horizontal axis gives the cutting edge wear in micrometres. Fig. 5 shows the force Fx which acts in the feed direction. The same dependence was found for all tested cutting edges. Increasing cutting edge wear on the clearance surface causes the increase of force load in the feed direction. During the first pass, the highest force Fx was reached by the cutting tools with cutting edge radius $25 \mu \mathrm{m}$. The maximum force Fx was $434 \mathrm{~N}$.

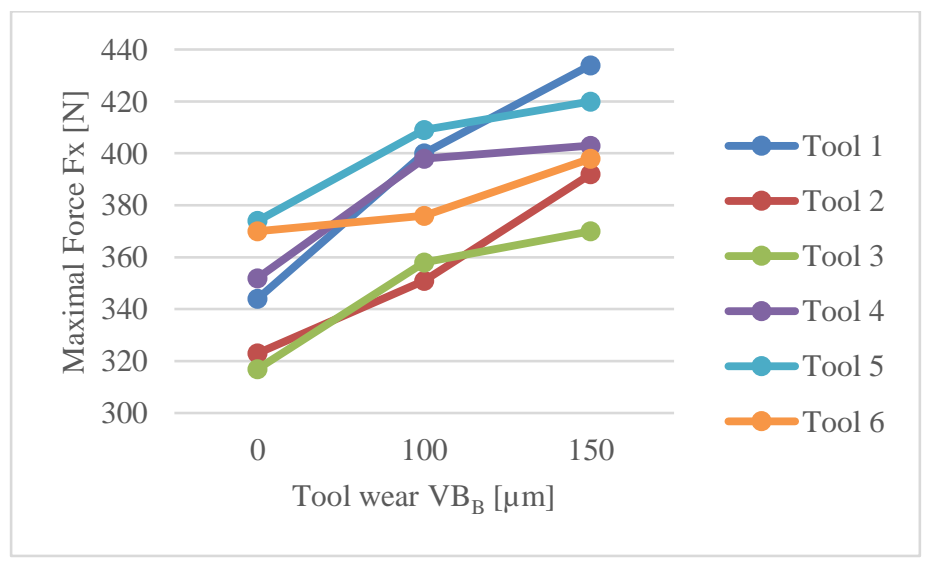

Fig. 5. Analysis of maximum Fx force 
Force Fy, which acts in the radial cut of depth, reached the highest value throughout the experiment. The mechanical properties of the machined alloy was the main reason for the highest Fy force values. As has already been mentioned, Inconel 718 has the property that after machining, the machined layer becomes stronger. Therefore, the cutting edge needs more energy for the following pass. Also the cutting edge needs to exert higher energy due to the physical law of action and reaction. The machined material, especially the machined layer pushes the cutting tool away from the machined surface during the machining process. The cutting edge radius $25 \mu \mathrm{m}$ also reaches the highest value in the radial cut of depth direction. It can be said, that the radius $25 \mu \mathrm{m}$ is high because of the force load results and comparison. In a nutshell, the cutting process needs higher energy for the plastic deformation, which is necessary for chip creation and transformation and this is undesirable.

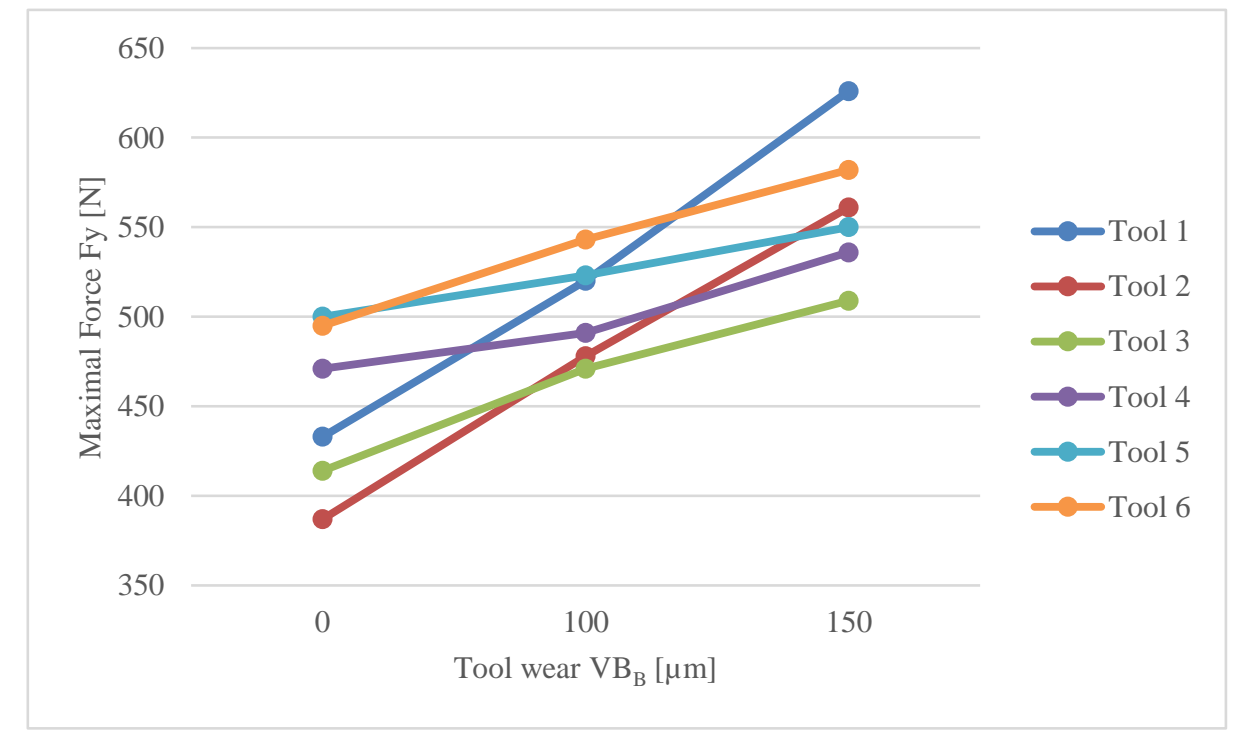

Fig. 6. Analysis of maximum Fy force

Last but not least, the forces in the direction of the cutting tool axis were evaluated. As you can see, the force load $\mathrm{Fz}$ was lower than forces Fx and Fy. Moreover, in this case, there was no dependence between the force load and tool wear for any of the tested cutting tools.

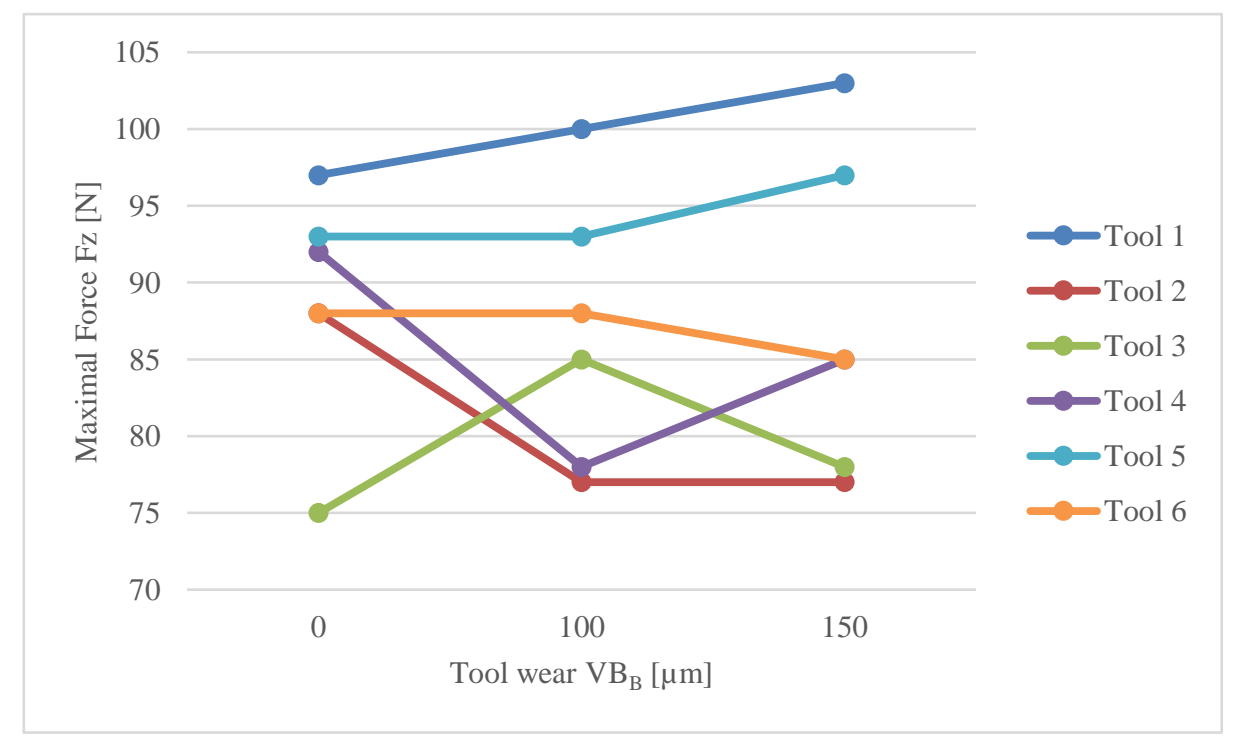

Fig. 7. Analysis of maximum Fz force

As has been said before, the results obtained the maximum force values. The following figure shows the change of the force load during one pass. It can be seen that the curves contain a large number of peaks. These peaks are caused by im-perfect homogeneity of the material, tool vibrations, balance, cutting edge geometry and rigidity of the CNC milling machine. All these parameters cause vibrations. Vibrations have a direct effect on the formation of these peaks. 


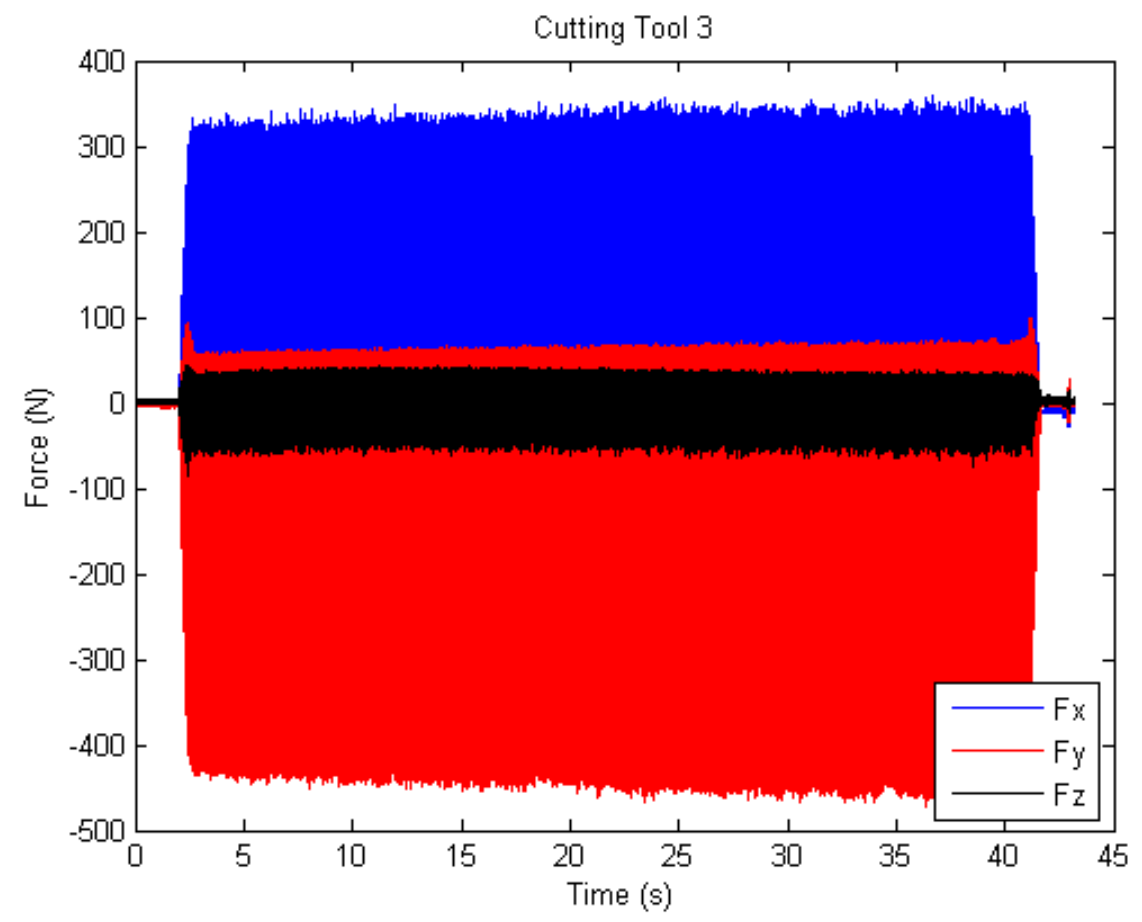

Fig. 8. Milling forces Fx; Fy; Fz

The median value of the force load was also investigated, because in every case, the maximum force load was higher than the median values $\left(\mathrm{x}_{\mathrm{s}}\right)$. The highest force was found in the radial depth of cut as can be seen from the previous results. Obviously, the resulting values of the forces were lower. Also the overall scattering of these dimensions has decreased. The same dependence between the value of the force load and the tool wear remains.

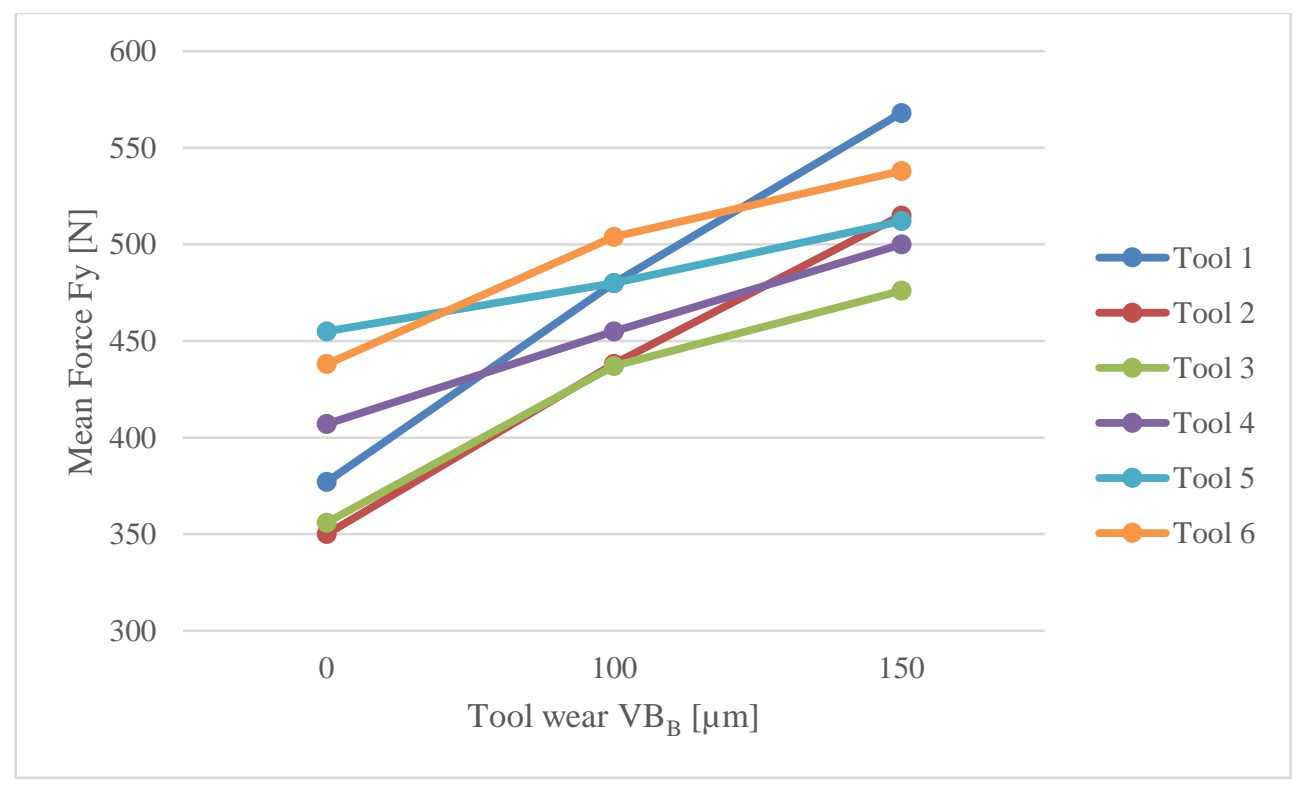

Fig. 9. Analysis of mean Fy force

\section{Conclusion}

High forces load causes a cutting tool wear in short time. Problem with force load was investigated in this article. The force loads in the direction of feed rate, radial depth of cut and spindle axis were measured when milling Inconel 718 , which is a hard machining alloy. These forces were evaluated depending on the actual cutting edge wear on the clearance surface. The force load was measured at $\mathrm{VB}_{\mathrm{B}}=0 \mu \mathrm{m}, \mathrm{VB}_{\mathrm{B}}=100 \mu \mathrm{m}$ a $\mathrm{VB}_{\mathrm{B}}=150 \mu \mathrm{m}$. A three-component static dynamometer was used for measuring the force load. 
The following conclusions are based on this experiment:

1. The highest force was obtained in the direction of radial cut depth when compared the Fx; Fy and Fz values were compared.

2. The high resistance of the machined material is the reason why the Fy values were highest during evaluations. This resistance is due to the properties of the machined alloy Inconel 718, especially the fact that the machined layer is strengthened.

3. Three variants of the cutting edge radius were used for the experiment. Tool 5 and 6 with cutting edge radius $25 \mu \mathrm{m}$ had the highest force in the direction Fx and Fy. Notch wear was created on the clearance surface of Tool 1 after a series of passes. This notching causes increased force loads.

4. Repetition was performed to ensure the reliability of the measured data. During the first pass, when the tool microgeometry was still retained, the following deviations of the Fy values were found: 4.1) Tool 1;2 . deviation $25 \mathrm{~N}$; 4.2) Tool 3; 4 - deviation $51 \mathrm{~N}$; 4.3) Tool 5; 6 -deviation $48 \mathrm{~N}$. These deviations can be considered acceptable.

5. In addition to measuring the maximum force value, the values of the mean force value $\left(\mathrm{x}_{\mathrm{s}}\right)$ were selected on the Fy force measurement. These values were on average $50 \mathrm{~N}$ less.

In the future, the influence of the microgeometry on force load will be investigated further. The aim of these experiments is to increase the quality of cutting process. Moreover, these experiments will find the relationships between cutting tool microgeometry, process parameters, the machined material and the surface roughness. The aim is also to increase the quality of cutting tools.

\section{Acknowledgments}

This paper includes results created within the project SGS-2016-005.

\section{References}

[1] Bolar, G.; Das, A. \& Joshi, S. N. (2018). Measurement and analysis of cutting force and product surface quality during end-milling of thin-wall components. Measurement, Vol. 121, pp. 190 - 204, ISSN: 0263 - 2241

[2] homel.vsb.cz [online]. [Accessed: 2018-10-1]. http://homel.vsb.cz/ cep77/PDF/EMO_kapitola_08.pdf

[3] Kataoka, R. \& Shamoto, E. (2018). Influence of vibration in cutting on tool flank wear: Fundamental study by conducting a cutting experiment with forced vibration in the depth-of-cut direction. Precision Engineering. ISSN: $0141-6359$

[4] Zhang, X.; Sui, H.; Zhang, D. \& Jiang. (2018). Measurement of ultrasonic-frequency repetitive impulse cutting force signal. Measurement, Vol. 129, pp. 653 - 663, ISSN: 0263 - 2241

[5] Baksa, T.; Schornik, V.; Adamek, P. \& Zetek, M. (2016). Machining of Inconel 718 Using Uncoated Cutting Tools with Different Cutting Edge Quality, Proccedings of the 27th DAAAM International Symposium, pp. 0441 - 0446, B. Katalinic (Ed.), Published by DAAAM International, ISBN 978-3-902734-08-2, ISSN 1726-9679, Vienna, Austria

[6] Denkena, D. \& Biermann, B. (2014). Cutting edge geometries. CIRP Annals - Manufacturing Technology, Vol. 63, pp. $631-653$, ISSN: $0007-8506$

[7] Aurich, J. C.; Effgen, Ch. \& Kirsch, B. (2016). Cutting edge preparation with elastin bonded superabrasive grinding wheels. CIRP Annals - Manufacturing Technology, Vol. 65, pp. 329 - 332, ISSN: 0007 - 8506

[8] Begmann, B. \& Grove, T. (2018). Basic principles for the design of cutting edge roundings. CIRP Annals Manufacutirng Technology, Vol. 67, pp. 73 - 78, ISSN: $0007-8506$ 